\title{
Anticoagulant Effects of Dabigatran in Paediatric Patients Compared with Adults: Combined Data from Three Paediatric Clinical Trials
}

\author{
Hugo Maas ${ }^{1}$ Savion Gropper ${ }^{2}$ Fenglei Huang ${ }^{3}$ Joachim Stangier ${ }^{1}$ Igor Tartakovsky ${ }^{2}$ \\ Martina Brueckmann ${ }^{2,4}$ Jacqueline M. L. Halton ${ }^{5}$ Lesley G. Mitchell ${ }^{6}$
}

${ }^{1}$ Translational Medicine and Clinical Pharmacology, Boehringer Ingelheim Pharma GmbH \& Co. KG, Biberach, Germany

Address for correspondence Lesley G. Mitchell, MSc, HMRC 405,

${ }^{2}$ Clinical Development and Medical Affairs, Boehringer Ingelheim University of Alberta, Edmonton, Alberta T6G 2S2, Canada International $\mathrm{GmbH}$, Ingelheim, Germany

${ }^{3}$ Translational Medicine and Clinical Pharmacology, Boehringer Ingelheim Pharmaceuticals, Inc., Ridgefield, Connecticut, United States

${ }^{4}$ Faculty of Medicine Mannheim, Department of Medicine I, University of Heidelberg, Mannheim, Germany

${ }^{5}$ Department of Pediatrics, Children's Hospital of Eastern Ontario, University of Ottawa, Ottawa, Ontario, Canada

${ }^{6}$ Division of Pediatric Hematology/Oncology, Department of Pediatrics, University of Alberta, Edmonton, Alberta, Canada

Thromb Haemost 2018;118:1625-1636.

Background Physiological age-related changes in the haemostatic and coagulation systems result in differing anticoagulant assay responses to standard anticoagulants. Therefore, we investigated the response of anticoagulant assays to dabigatran etexilate (DE) in children compared with adults.

Objective This article assesses the relationship between plasma dabigatran concentration and coagulation assay results across age groups in children and adults.

Patients and Methods Data from three clinical trials in which children received DE following standard of care for venous thromboembolism were compared with data from adult clinical trials. The effects of dabigatran concentration on diluted thrombin time (dTT), ecarin clotting time (ECT) and activated partial thromboplastin time (aPTT) were analysed graphically and with modelling.

Results The concentration-dTT relationships were consistent in children across all ages and adults in the graphical analysis. For ECT and aPTT, relationships based on ratios over baseline were similar across all ages; absolute clotting times showed that the same exposure resulted in longer clotting times in some of the children aged $<1$ year versus adults. Modelling showed concentration-clotting time relationships for all three assays were largely comparable between adults and children, except in those aged $<2$ months, in whom there was a slight upward shift in ECT and aPTT relative to adults.

Conclusion Results suggest that developmental haemostatic changes will have little impact on response to DE. However, further paediatric clinical trials assessing the relationship between coagulation assay responses and clinical outcomes will be needed to confirm this finding. received

January 22, 2018

accepted after revision June 29, 2018
DOI https://doi.org/

$10.1055 / \mathrm{s}-0038-1668132$. ISSN 0340-6245. (c) 2018 Georg Thieme Verlag KG Stuttgart . New York
License terms

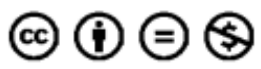




\section{Introduction}

The overall annual incidence of venous thromboembolism (VTE) in the paediatric population is approximately 0.07 to 0.14 events per 10,000 children, but this number is increasing. ${ }^{1-3}$ The current standard of care (SOC) in paediatric VTE is unfractionated heparin (UFH) or low molecular weight heparin (LMWH) followed by UFH, LMWH, or vitamin $\mathrm{K}$ antagonists (VKAs). ${ }^{4-6}$ However, all of these agents have limitations, such as parenteral administration in the case of heparins and drug-drug and drug-food interactions, and the need for coagulation monitoring in the case of VKAs. ${ }^{4,6,7}$ Dabigatran, a direct thrombin inhibitor, which is administered as the orally available pro-drug dabigatran etexilate (DE), has demonstrated efficacy and safety for use in the prevention and treatment of VTE in adults and may overcome some of the limitations associated with current SOC in children. ${ }^{8-10}$

Throughout childhood, physiological age-related changes occur in the haemostatic and coagulation systems that alter coagulation assay responses to standard anticoagulant treatments such as standard heparin, LMWH and VKA. ${ }^{11,12}$ Most pro- and anticoagulation factors, for example, are decreased in children, with differences most pronounced in those aged $<12$ months when compared with older children and adults. ${ }^{5,6,13,14}$ In addition, most cases of VTE in children are secondary to severe underlying disorders, which can result in acquired abnormalities in the haemostatic system. ${ }^{1}$ Therefore, studies to assess the relationship between anticoagulant concentration and effect on coagulation assay clotting times in the paediatric population are necessary, and have been used to demonstrate the differences between children and adults for anticoagulants, including UFH, LMWH and VKA. ${ }^{15-17}$

The anticoagulant effect of dabigatran across paediatric age groups was assessed in an in vitro study that used pooled plasma samples from healthy children. When corrected for baseline, the clotting time responses to dabigatran in children were comparable between all age groups. They were also similar to those in adults, either for the untransformed clotting times or after correction as a ratio to baseline values, depending on the assay used. ${ }^{18}$ As in adults with VTE, different assays respond differently to dabigatran when used in children: a linear concentration-response relationship was observed for ecarin clotting time (ECT) and diluted thrombin time (dTT), whereas a non-linear relationship was seen for activated partial thromboplastin time (aPTT), in both the previously mentioned in vitro study and in clinical studies in paediatric patients with VTE. ${ }^{18-20}$

The current post hoc analysis was conducted to compare the relationship between plasma dabigatran concentration and clotting times, as measured with dTT, ECT and aPTT assays ex vivo in paediatric patients being treated for VTE in three phase II studies and to evaluate the effect of age on the relationship between plasma dabigatran concentration and coagulation assay results, specifically in those under 2 years of age. For comparison, data observed in adult volunteers and adults with VTE are reported.

\section{Materials and Methods}

The current analysis used data from three clinical trials of dabigatran conducted in children with VTE and four clinical trials of dabigatran conducted in adults, either adults with VTE or healthy adult volunteers. These trials were selected because they included the pharmacodynamic (PD) endpoints $\mathrm{dTT}, \mathrm{ECT}$ and aPTT. The dTT assays were performed to provide an estimate of the concentration of dabigatran.

The three paediatric trials were: study 1160.88 (NCT00844415; patients aged 12 to $<18$ years), study 1160.89 (NCT01083732; patients aged 1 to $<12$ years) and study 1160.105 (NCT02223260; patients aged $<1$ year), details of which have previously been reported. ${ }^{20-22}$ Study 1160.88 was a phase Ila, open-label, multi-centre, single-arm trial to evaluate the safety and tolerability and assess preliminary pharmacokinetic (PK) and PD parameters of dabigatran in adolescent patients, aged between 12 and $<18$ years. After successfully completing treatment with LMWH or oral anticoagulation for primary VTE, patients received weightadjusted doses of DE administered as capsules twice daily for 3 days. Initially, patients received $1.71( \pm 10 \%) \mathrm{mg} / \mathrm{kg}$ ( $80 \%$ of the adult dose of $150 \mathrm{mg} / 70 \mathrm{~kg}$ twice daily, adjusted for weight). Subsequently, patients received $2.14( \pm 10 \%) \mathrm{mg} / \mathrm{kg}$ (100\% of the adult dose adjusted per patient weight). ${ }^{20}$ Study 1160.89 was a phase Ila, open-label, multi-centre, single-arm trial to evaluate the safety, tolerability, PK and PD of an oral liquid formation of DE in patients aged 1 to $<12$ years. Having completed planned treatment with LMWH or oral anticoagulants for VTE, patients received a single weight and ageadjusted dose of DE (equivalent to $150 \mathrm{mg}$ twice daily in adults). Originally, all children were to receive weight-adjusted doses, twice daily for 3 days. However, this was replaced with a single age- and weight-adjusted dose during the course of the study. ${ }^{21}$ Study 1160.105 was a phase Ila, open-label, multicentre, single-arm trial to assess the PK/PD relationship, safety and tolerability of DE in infants aged $<1$ year, who had completed standard anticoagulant treatment for VTE. Infants received a single age- and weight-adjusted dose of $\mathrm{DE}$, administered as an oral liquid formulation. ${ }^{22}$ The algorithms used to determine the age-/weight-adjusted dosing in the three paediatric studies differed, due to modifications over time.

Data from two adult populations were compared with those of the paediatric groups. A summary of population characteristics is provided in -Table $\mathbf{1}$.

One population, referred to as "adult volunteers," comprised healthy subjects who took part in the phase I study, 1321.1 (NCT016888302) 23 and healthy subjects or those with mild or moderate renal impairment who took part in the phase I study, 1321.2 (NCT01955720). ${ }^{24}$ Study 1321.1 was a randomized, placebo-controlled, double-blind, proof-ofconcept trial to assess the safety, tolerability and efficacy of idarucizumab for the reversal of the anticoagulant effects of dabigatran. Healthy adult volunteers all received DE $220 \mathrm{mg}$ twice daily for 3 days and a final dose on day 4 , followed by idarucizumab or placebo. ${ }^{23}$ Study 1321.2 was a randomized, double-blind, crossover study in which middleaged, elderly and renally impaired subjects received DE 220 
Table 1 Variables from the adult studies

\begin{tabular}{|c|c|c|c|c|}
\hline & \multicolumn{2}{|l|}{ Adult volunteer } & \multicolumn{2}{|l|}{ Adult patient } \\
\hline & $\begin{array}{l}\text { Study } 1321.1 \\
\text { (NCT01688830) }^{a}\end{array}$ & $\begin{array}{l}\text { Study } 1321.2 \\
(\text { NCT01955720) }\end{array}$ & $\begin{array}{l}\text { Study } 1160.53 \\
\text { (RE-COVER; } \\
\text { NCT00291330) }\end{array}$ & $\begin{array}{l}\text { Study } 1160.64 \\
\text { (RE-NOVATE II; } \\
\text { NCT00657150) }\end{array}$ \\
\hline Number of patients in the PK set & 51 & 46 & 1,179 & 702 \\
\hline Age mean, y (range) & $31(20-45)$ & $64(45-76)$ & $55(18-93)$ & $61(23-87)$ \\
\hline Dosing regimen (s) & $220 \mathrm{mg}$ bid & $\begin{array}{l}150 \text { mg bid }{ }^{\mathrm{b}} \text {, } \\
220 \mathrm{mg} \text { bid }\end{array}$ & $150 \mathrm{mg}$ bid & $220 \mathrm{mg} \mathrm{qd}$ \\
\hline Dabigatran concentration ${ }^{\mathrm{C}} \mathrm{IQR}, \mathrm{ng} / \mathrm{mL}$ & $67.4-181$ & $66.4-218$ & $37.3-102$ & $17.2-91.9$ \\
\hline \multicolumn{5}{|l|}{$\mathrm{dTT}$} \\
\hline Number of observations & 739 & 793 & $-{ }^{d}$ & 1,940 \\
\hline Baseline mean, s (range) & $32.1(29.4-38.1)$ & $32.1(29.7-38.1)$ & - & $32.1(11.4-82.6)$ \\
\hline \multicolumn{5}{|l|}{ ECT } \\
\hline Number of observations & 739 & 793 & 2,285 & $-{ }^{e}$ \\
\hline Baseline mean, s (range) & $37.4(33.6-42.5)$ & $34.6(30.3-38.9)$ & $-{ }^{f}$ & - \\
\hline \multicolumn{5}{|l|}{ aPTT } \\
\hline Number of observations & 737 & 792 & 2,278 & 1,933 \\
\hline Baseline mean, s (range) & $33.2(23.5-40.0)$ & $29.7(22.1-38.4)$ & $-{ }^{f}$ & $32.6(19.6-156)$ \\
\hline
\end{tabular}

Abbreviations: aPTT, activated partial thromboplastin time; bid, twice daily; dTT, diluted thrombin time; ECT, ecarin clotting time; IQR, interquartile range; PK, pharmacokinetic; qd, once daily.

Note: Summary of dabigatran concentration and clotting assay variables for the adult volunteer and adult patient studies. The following measurements were excluded due to implausibly high values: study 1160.53, aPTT measurements at visit 4 in two patients and at visit 9 in one patient; study 1321.1, aPTT measurement at visit 2 for one patient; study 1321.2, aPTT, ECT and dTT measurements for one patient at visit 3 and aPTT measurement for a second patient at visit 3 .

${ }^{a}$ Only placebo and dabigatran treatment periods were used for the current analysis.

bSubjects with mild or moderate renal impairment received $150 \mathrm{mg}$ bid.

'Based on plasma concentration samples across all sampling times, including samples measured at times other than trough (pre-dose) and samples taken outside of the sampling window.

${ }^{\mathrm{d}}$ No dTT was measured in study 1160.53 .

${ }^{\mathrm{e}}$ No ECT was measured in study 1160.64 .

${ }^{f}$ No baseline measurements were taken in study 1160.53 .

or $150 \mathrm{mg}$ twice daily for 4 days, followed by idarucizumab or placebo. ${ }^{24}$ Only data obtained during the placebo and dabigatran-only treatment periods of study 1321.1 and study 1321.2 were used in the current analysis.

The second adult population, referred to as "adult patients," comprised patients from the double-blind, randomized, multi-centre RE-COVER study (study 1160.53; NCT00291330), which compared the efficacy and safety of 6 months of treatment with DE $150 \mathrm{mg}$ twice daily with dose-adjusted warfarin for acute symptomatic VTE. ${ }^{8}$ Because no dTT or baseline measurements were taken in this study, data from patients of a similar age who were treated in the randomized, double-blind RE-NOVATE II trial (study 1160.64; NCT00657150) $)^{25}$ were used when necessary. RENOVATE II compared the efficacy and safety of DE $220 \mathrm{mg}$ once daily with enoxaparin $40 \mathrm{mg}$ once daily for VTE prevention following elective hip replacement surgery. ${ }^{25}$ Descriptions of these adult volunteer and adult patient studies have previously been reported in detail. $8,23-25$

Demographics and clotting assay variables for the adult and paediatric studies are shown in - Tables $\mathbf{1}$ and $\mathbf{2}$, respectively.
Clotting time measurements from samples taken from all paediatric, healthy volunteers and adult patients were obtained from one central laboratory (menal GmbH, Emmendingen, Germany), which minimized variability between assay methods and laboratories.

\section{Assays}

The following assays were each performed using 10-channel coagulometers (MERLIN medical MC10PLUS).

Diluted Thrombin Time The plasma sample $(50 \mu \mathrm{L})$ was diluted with $0.9 \%$ sodium chloride $(350 \mu \mathrm{L}$ ). A total of $50 \mu \mathrm{L}$ of the diluted sample was then combined with $100 \mu \mathrm{L}$ of normal pooled human plasma and incubated for 2 minutes at $37^{\circ} \mathrm{C}$. Clotting was initiated with the addition of $100 \mu \mathrm{L}$ of human calcium thrombin. The time between the addition of the thrombin to clot formation was recorded as the coagulation time.

Ecarin Clotting Time The plasma sample $(75 \mu \mathrm{L})$ was combined with $75 \mu \mathrm{L}$ of imidazole/veronal buffer and incubated for 2 minutes at $37^{\circ} \mathrm{C}$. The clotting measurement was initiated with the addition of $75 \mu \mathrm{L}$ of ecarin solution; the time taken for clot formation was recorded as the ECT. 
Table 2 Variables from the paediatric studies

\begin{tabular}{|c|c|c|c|c|}
\hline & $\begin{array}{l}\text { Study } 1160.88 \\
\text { Ages } 12 \text { to }<18 \text { y } \\
\text { (NCT00844415) }\end{array}$ & $\begin{array}{l}\text { Study } 1160.89 \\
\text { Ages } 1 \text { to }<12 \text { y } \\
\text { (NCT01083732) }\end{array}$ & $\begin{array}{l}\text { Study } 1160.105 \\
\text { Ages < } 1 \text { y } \\
\text { (NCT02223260) }\end{array}$ & $\begin{array}{l}\text { From study } 1160.105 \\
\text { and subset from } \\
\text { study } 1160.89 \\
\text { Ages }<2 \text { y }\end{array}$ \\
\hline Number of patients in the PK set & 9 & $18^{a}$ & 8 & 14 \\
\hline Age $^{\mathrm{b}}$ mean (range) & $16.2(13.8-18.0) y$ & $4.8(1.2-11.8) y$ & $2.4(1-5) \mathrm{mo}$ & $8.8(1-23) \mathrm{mo}$ \\
\hline Dabigatran concentration ${ }^{\mathrm{C}} \mathrm{IQR}, \mathrm{ng} / \mathrm{mL}$ & $38.2-75.7$ & $42.5-107$ & $60.1-128$ & $54.9-121$ \\
\hline \multicolumn{5}{|l|}{$\mathrm{dTT}$} \\
\hline Number of observations & 42 & 56 & 24 & 42 \\
\hline Baseline mean, s (range) & $30.4(27.3-35.8)$ & $34.0(29.8-40.2)$ & $30.6(27.8-32.7)$ & $31.2(27.8-33.7)$ \\
\hline \multicolumn{5}{|l|}{ ECT } \\
\hline Number of observations & 42 & 38 & 24 & 41 \\
\hline Baseline mean, s (range) & $33.8(30.1-39.2)$ & $36.9(31.7-43.4)$ & $40.7(35.7-47.3)$ & $39.1(33.4-47.3)$ \\
\hline \multicolumn{5}{|l|}{ aPTT } \\
\hline Number of observations & 41 & 42 & 24 & 41 \\
\hline Baseline mean, s (range) & $34.7(27.5-53.4)$ & $33.7(19.8-44.8)$ & $42.1(36.0-51.2)$ & $37.9(19.8-51.2)$ \\
\hline
\end{tabular}

Abbreviations: aPTT, activated partial thromboplastin time; dTT, diluted thrombin time; ECT, ecarin clotting time; IQR, interquartile range; PK, pharmacokinetic.

Note: Summary of dabigatran concentration and clotting assay variables for the paediatric studies (the subset of patients aged $<2$ years is combined from studies 1160.89 and 1160.105). The following measurements were excluded for being outliers: study 1160.88 , aPTT at 2 hours for one patient; study 1160.89, aPTT and ECT at 10 hours for two patients, baseline aPTT and ECT for a third patient and baseline aPTT for a fourth patient.

${ }^{a}$ Three receiving multiple-dose treatment, 15 receiving single-dose treatment.

${ }^{\mathrm{b}}$ Age at time of (first) dose administration, rounded to 1 decimal.

'Based on plasma samples across all sampling times, including samples measured at times other than trough (pre-dose) and samples taken outside of the sampling window.

Activated Partial Thromboplastin Time The plasma sample $(75 \mu \mathrm{L})$ was combined with $75 \mu \mathrm{L}$ of kaolin-cephalin reagent and incubated for 3 minutes at $37^{\circ} \mathrm{C}$ with continuous stirring, prior to the addition of $75 \mu \mathrm{L}$ of $0.025 \mathrm{M}$ calcium chloride, which initiated clotting. The time from the addition of calcium chloride to the formation of a fibrin clot was measured as aPTT.

\section{Graphical and Modelling Analyses}

For each of the three paediatric studies, graphs showing plasma dabigatran concentrations against dTT, ECT and aPTT coagulation times were superimposed on to those from adult volunteers and adult patients. Dabigatran concentration-clotting time observations were expressed untransformed and in terms of their ratio over baseline, if baseline data were available.

The relationships between dabigatran concentrations and clotting times in paediatric and adult datasets were subsequently characterized using modelling. The purpose of this was twofold: (1) the model-based characterization allowed a closer evaluation of the effect of age on the expected range of clotting parameters in the paediatric population; and (2) it allowed adult ranges to be calculated for concentration-clotting time relationship parameters, which could serve as a reference for the comparison with patients in the paediatric dataset.

To achieve these objectives, the modelling approach had to provide predictions on an individual subject-level. Therefore, 'mixed-effects' or 'population' models ${ }^{26}$ were developed that describe the concentration-clotting time relationship both on a population mean level and in terms of interindividual variability.

Linear relationships were fitted to subjects' concentration-dTT and concentration-ECT profiles. To describe interindividual variability, intercept and slope parameters were considered random variables with variability terms proportional to the parameters' typical values. The relationship between concentration and aPTT was modelled similarly, except a non-linear 'Emax' relationship was assumed rather than a linear relationship. ${ }^{26,27}$ The 'Emax' model describes aPTT in terms of a baseline effect ('EO') and a dabigatran effect, which is a hyperbolic function of drug concentration. The dabigatran effect is described by a maximum effect parameter ('Emax') and a potency parameter ('EC50'), which represents the dabigatran concentration at the half-maximal effect. In order for all parameters in the model to remain estimable, inter-individual variability terms were incorporated on the 'E0' and 'Emax'” parameters only.

The linear and non-linear concentration-clotting time models were applied to the complete paediatric data and to the adult volunteer dataset. The data in these sets were expected to be sufficiently informative to allow estimation of parameters, including inter-individual variability.

Modelling outputs included subject-specific estimates of intercept and slope parameters (for the linear dTT and ECT models) and baseline and maximum effect (for the non-linear 
aPTT model). The estimates obtained for the adult population were summarized into reference ranges (minimum, maximum values), whereas those for paediatric patients were regressed non-parametrically against age. Regressions were performed separately for paediatric patients aged $<2$ years and those aged $\geq 2$ years.

Model development was guided by numerical criteria and basic graphical goodness-of-fit diagnostics. Numerical criteria included objective function value, condition number, relative standard errors and shrinkage estimates. Graphical diagnostics included residual plots and plots of observed versus predicted clotting times.

\section{Software}

Mixed-effects modelling was performed using NONMEM version 7.3 (Icon Development Solutions, Ellicott City, Maryland, United States). Data preparation, graphical summaries and non-parametric regressions of dabigatran concentration-clotting time parameters against age were performed using the R statistical environment version 3.0.3 (R Foundation for Statistical Computing, Vienna, Austria).

\section{Results}

\section{Graphical Analysis}

Diluted Thrombin Time Superimposing data from each paediatric study on to results from adult volunteers ( - Fig. 1A, top row: untransformed dTT, bottom row: dTT ratio over baseline) and adult patients ( $\mathbf{F i g}$. 1B, top row: untransformed dTT, bottom row: dTT ratio over baseline) showed that paediatric concentration-dTT profiles were consistent with those observed in both adult populations. Note that because dTT data were unavailable from study 1160.53 (treatment of acute VTE), adult patient data were all taken from study 1160.64 (primary VTE prevention in patients undergoing total hip replacement surgery).

Ecarin Clotting Time For ECT, dabigatran concentrationclotting time relationships based on ratios over baseline were similar across all age groups (bottom row of - Fig. 2A). Note

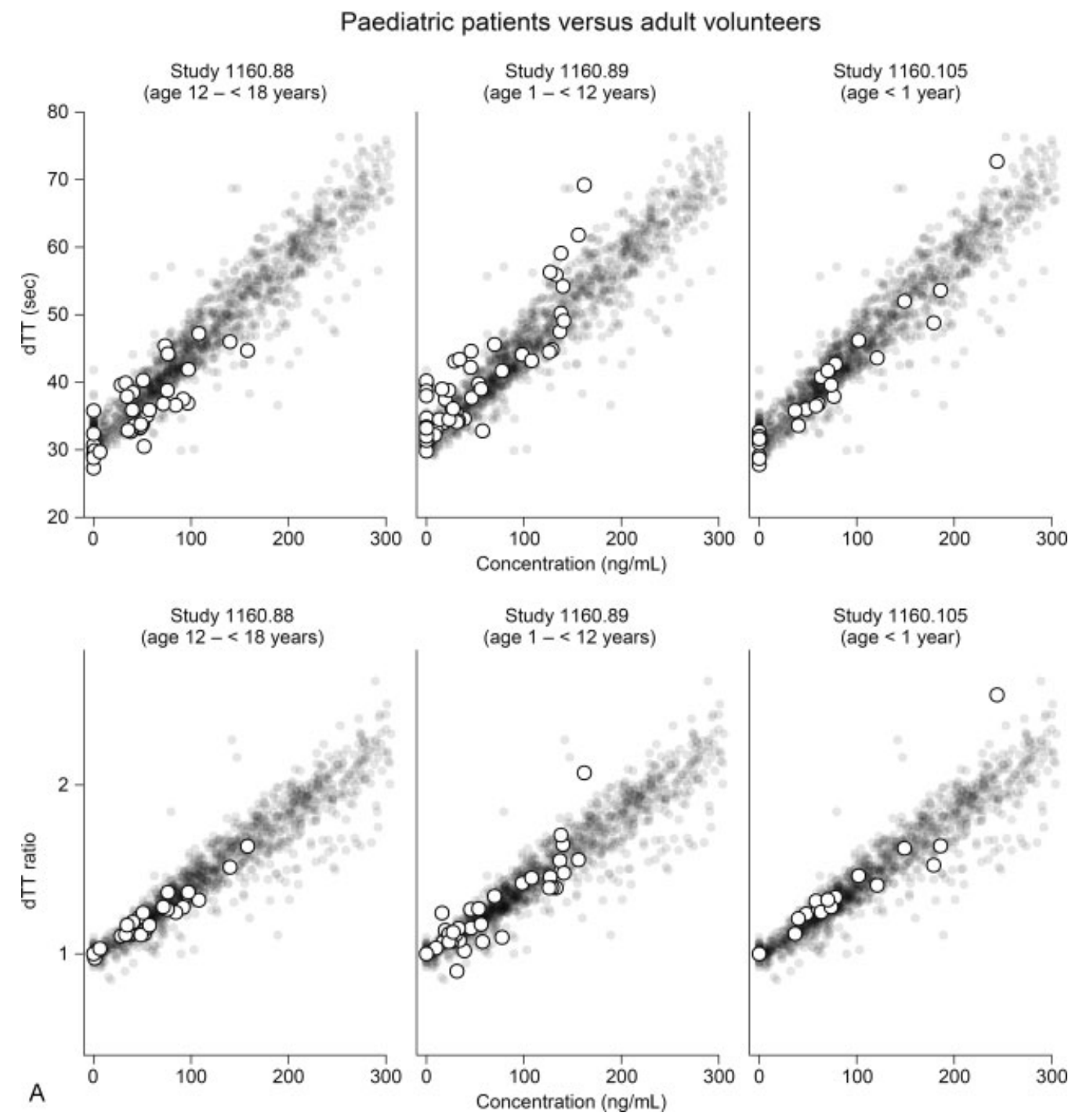

Fig. 1 Concentration-diluted thrombin time (dTT) relationships. Concentration-dTT relationships for paediatric patients (white circles) overlaid on those of (A) adult volunteers (grey circles) or (B) adult patients (grey circles), for each of the three paediatric studies. Top rows of (A) and (B) show untransformed dTT values; bottom rows of (A) and (B) show dTT expressed as a ratio over baseline. In (B), adult patient data are taken from study 1160.64 . 

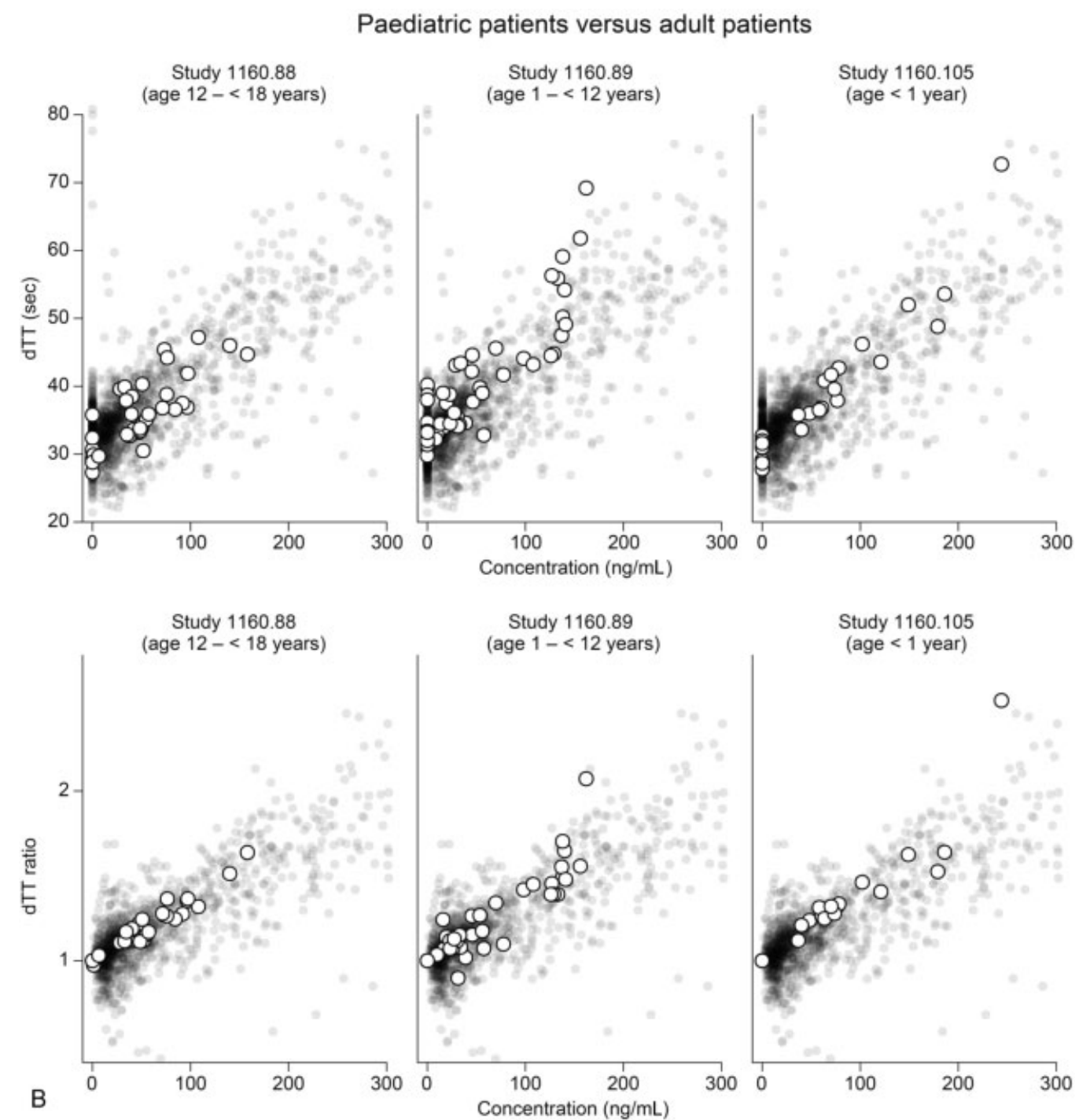

Fig. 1 (Continued)

that ECT ratios were not derived for adult patients as baseline data were not available. Data based on absolute clotting times ( - Fig. 2B and top row of - Fig. 2A), showed concentration-ECT profiles were shifted upwards for patients aged $<1$ year compared with the adult groups, which means that the same exposure resulted in longer clotting times. The concentration-clotting time relationship was also shifted upwards in those aged 1 to $<12$ years compared with adult patients, but not adult volunteers. This observation implies that concentration-ECT profiles differed between the two comparator adult groups (adult volunteers [-Fig. 2A] and adult patients [-Fig. 2B]).

Activated Partial Thromboplastin Time For aPTT, dabigatran concentration-clotting time relationships based on ratios over baseline were also similar across all age groups (bottom rows of - Figs. 3A and B). Study 1160.64, rather than study 1160.53 , was used to provide ratios over baseline due to the unavailability of baseline data. As for ECT, data based on absolute clotting times (top rows of - Figs. $3 \mathbf{A}$ and $\mathbf{B}$ ), showed concentration-aPTT profiles were shifted upwards for patients aged $<1$ year compared with the adult groups.

\section{Modelling Analysis}

Diluted Thrombin Time -Supplementary Table S1 summarizes parameter estimates for the modelled concentrationdTT relationships in the two populations (paediatric patients and adult volunteers). When the individual-patient parameter estimates were plotted against age (-Supplementary Fig. S1A, available in the online version), they were approximately constant across the age range for patients aged $<2$ years and their means fell within the adult ranges. For paediatric patients aged $>2$ years, parameters were lower in adolescents than in those aged 2 to $<12$ years; mean predicted parameters fell within adult ranges, except for the mean of the intercept parameter (corresponding to baseline clotting time) in those aged 15 to $<18$ years, which was close to or below the adult lower value.

Ecarin Clotting Time - Supplementary Table S2 summarizes parameter estimates for the modelled concentrationECT relationships in the two populations (paediatric patients and adult volunteers). When the individual-patient parameter estimates were plotted against age (-Supplementary Fig. S1B, available in the online version), they were within adult ranges 

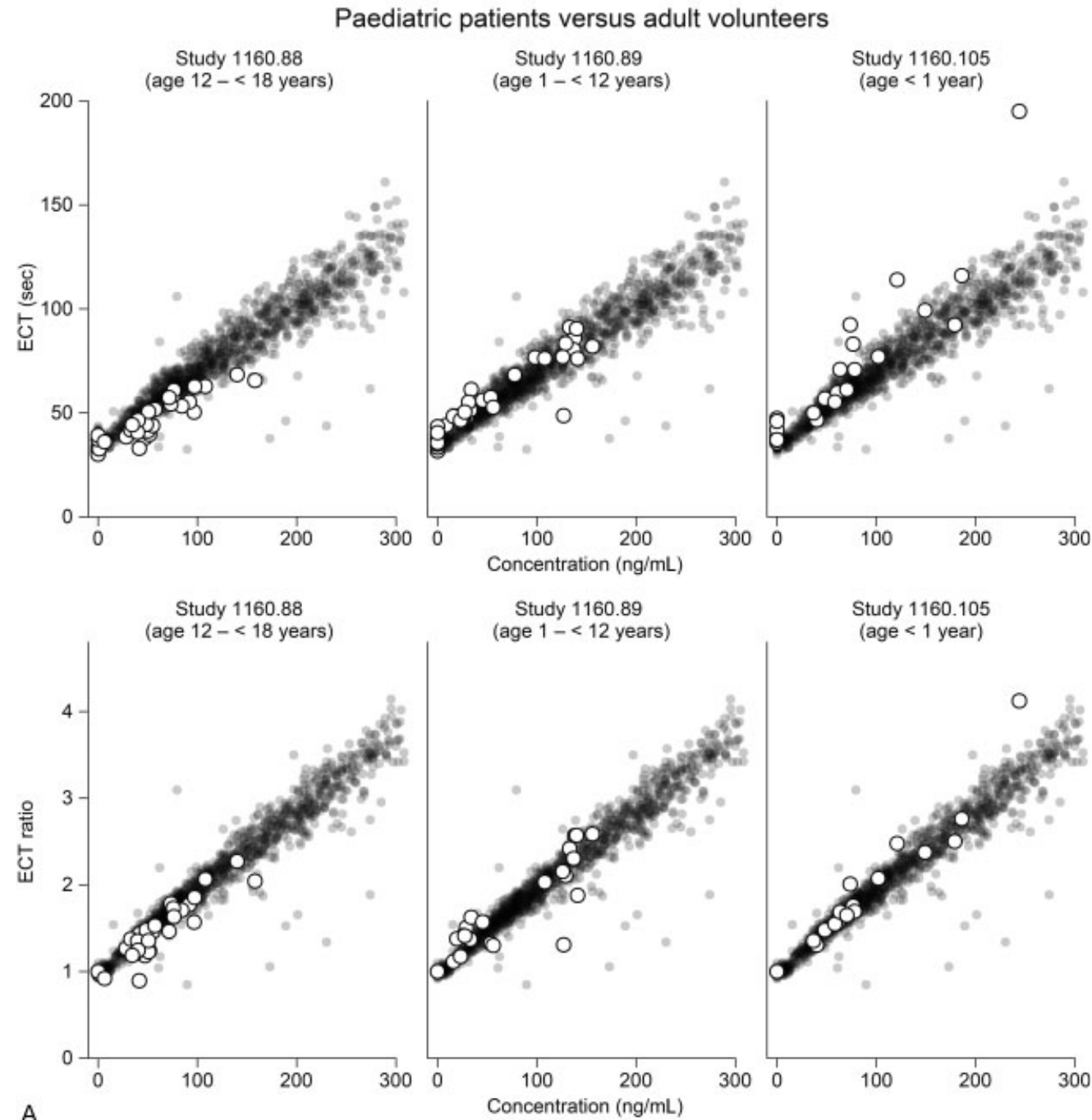

A

Paediatric patients versus adult patients
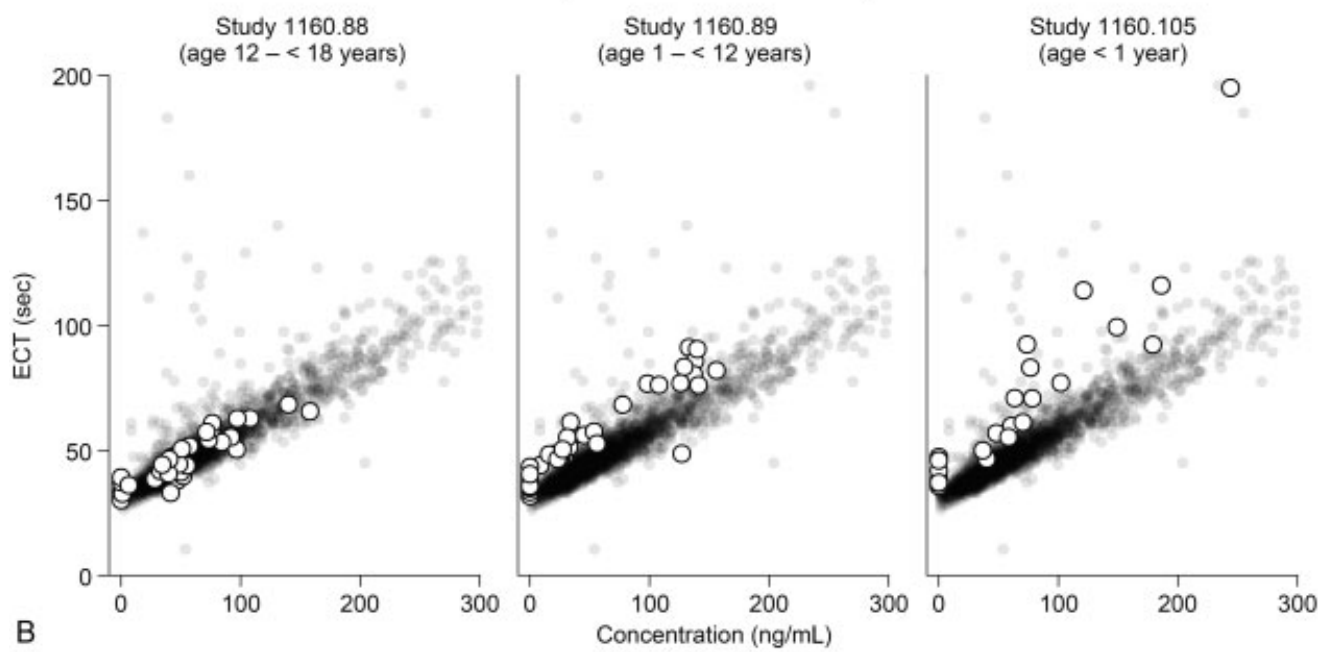

Fig. 2 Concentration-ecarin clotting time (ECT) relationships. Concentration-ECT relationships for paediatric patients (white circles) overlaid on those of (A) adult volunteers (grey circles) and (B) adult patients (grey circles), for each of the three paediatric studies. Top row of (A) shows untransformed ECT values; bottom row of (A) shows ECT expressed as a ratio over baseline. In (B), only untransformed ECT values are shown; adult patient data are from study 1160.53 . 
for paediatric patients aged $<2$ years, except in those aged $<2$ months ( $n=4$ subjects overall), for whom they were higher (in these patients ECT values were increased by $\sim 10-15 \%$ for dabigatran concentrations of $0-250 \mathrm{ng} / \mathrm{mL}$, respectively, compared with the most sensitive concentration-ECT profile predicted in adult volunteers). In paediatric patients aged $>2$ years, parameters were slightly lower in adolescent patients than in those aged 2 to $<12$ years. However, mean predicted parameters were within the adult ranges, except for the slope parameter (corresponding to drug effect on clotting time) for patients aged 15 to $<18$ years, which fell below the adult lower limit (-Supplementary Fig. S1B, available in the online version).

Activated Partial Thromboplastin Time - Supplementary Table $\mathbf{3} 3$ summarizes the parameter estimates for the modelled concentration-aPTT relationships in the two populations (paediatric patients and adult volunteers). When the individual-patient parameter estimates were plotted against age
(-Supplementary Fig. S1C, available in the online version), they were within the adult ranges for patients aged $<2$ years, with the exception of those aged $<2$ months $(n=4)$ for whom they were higher (in these patients aPTT values were increased by $\sim 10-20 \%$ for dabigatran concentrations of $0-250 \mathrm{ng} / \mathrm{mL}$, respectively, compared with the most sensitive concentrationaPTT profile predicted in adult volunteers). In paediatric patients aged $>2$ years, parameter estimates varied and were on average in the higher end of the adult range (-Supplementary Fig. S1C, available in the online version).

\section{Discussion}

The purpose of the current study was to assess the effect of age on the relationship between plasma dabigatran concentration and standard coagulation assay results, which may help to determine the most appropriate assay for paediatric use. Children aged $<2$ years were of particular interest, and

\section{Paediatric patients versus adult volunteers}
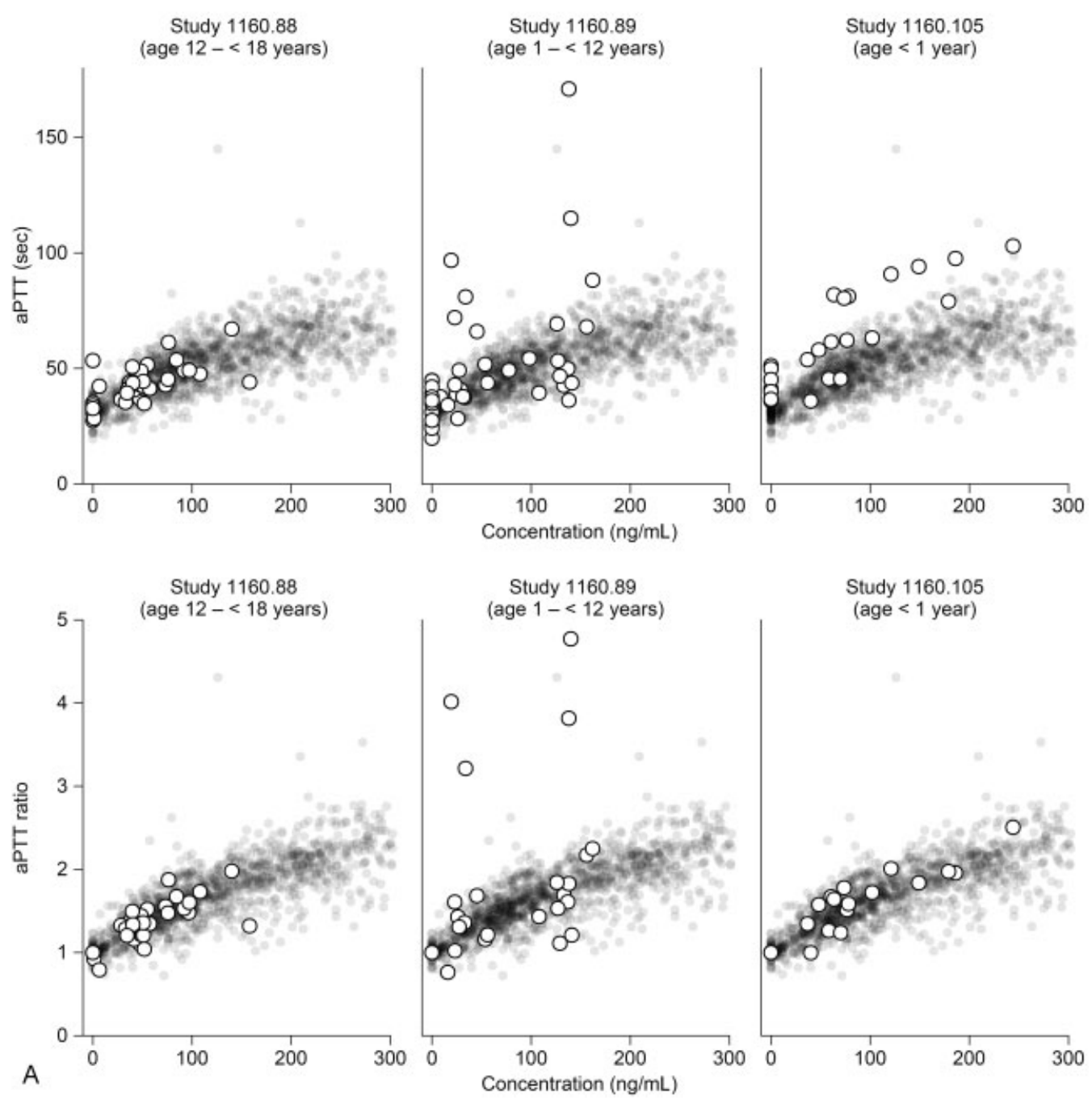

Fig. 3 Concentration-activated partial thromboplastin time (aPTT) relationships. Concentration-aPTT relationships for paediatric patients (white circles) overlaid on those of (A) adult volunteers (grey circles) and (B) adult patients (grey circles), for each of the three paediatric studies. Top rows of (A) and (B) show untransformed aPTT values; bottom rows of (A) and (B) show aPTT expressed as a ratio over baseline. In (B), adult patient data for untransformed coagulation times were taken from study 1160.53; for ratios over baseline, adult patient data were taken from study 1160.64. 


\section{Paediatric patients versus adult patients}
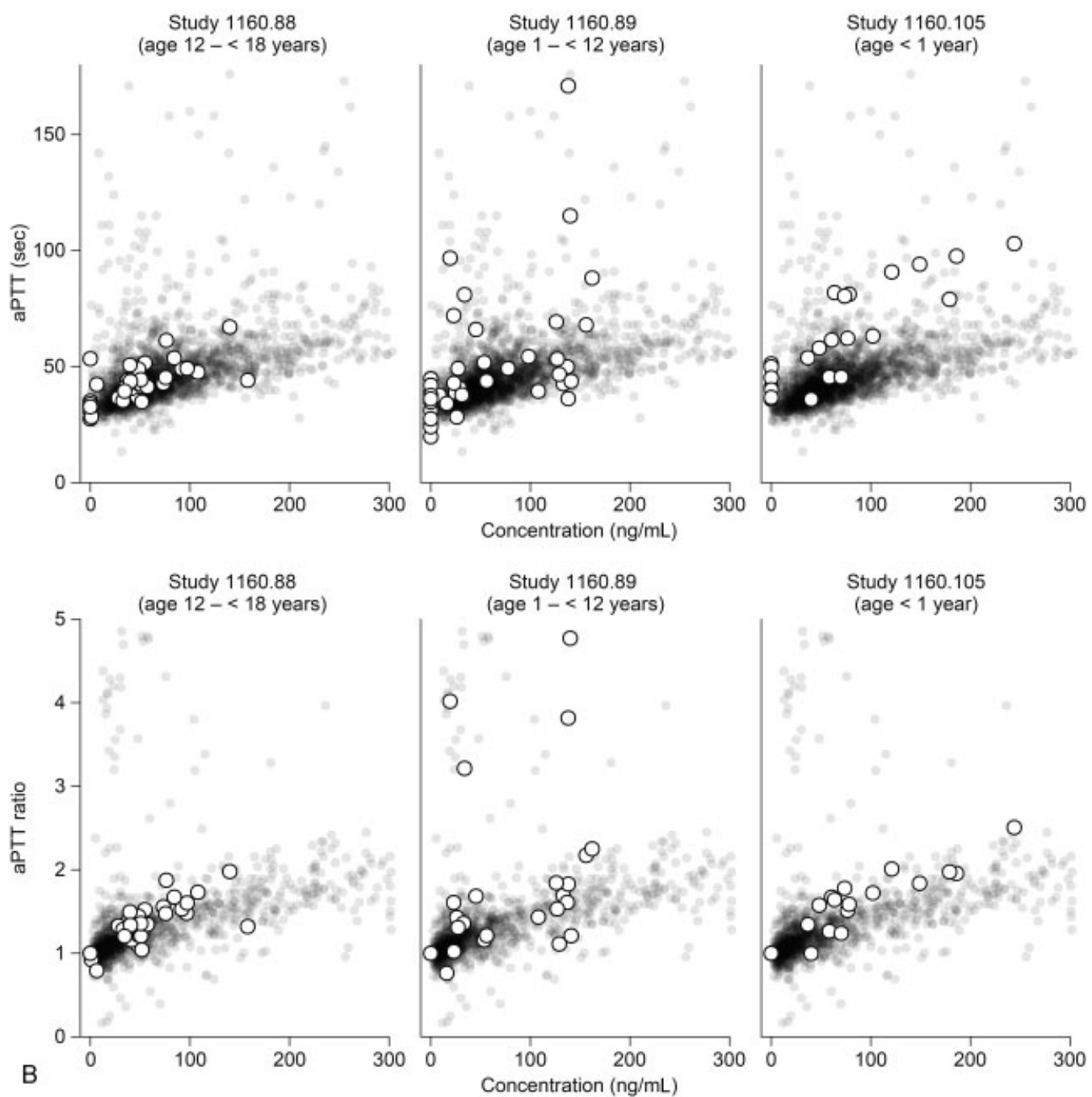

Fig. 3 (Continued)

assessed separately in the modelling analysis, as age-related differences in the haemostatic and coagulation system are most pronounced in very young children and therefore may warrant special attention. ${ }^{14}$ Findings from the graphical and modelling analyses indicated that concentration-dTT relationships were similar across age groups. Compared with adults, aPTT and ECT slightly increased in children aged $<1$ year according to the results of the graphical analysis (based on absolute clotting time), and in those aged $<2$ months based on the modelling analysis.

The shapes of the plots of individual-patient parameter estimates versus dabigatran concentrations ( - Supplementary Fig. S1, available in the online version) reflect how baseline clotting time and drug effect on clotting time vary across different age groups. For example, the variability of parameters is higher in children $<2$ years, and there is a downward trend by age for all three assays (even though all parameters are mostly within adult reference limits). In addition, while mean estimates are increased compared with adults for ECT and aPTT in the youngest infants and aPTT in toddlers and school children, they are below adult ranges in adolescents. These variations may be due to normal age-related physiological changes or other factors that may differ according to age, such as co-morbidities and comedications. As each study recruited patients from a different age range, the shape of the curves reflects, to some extent, between-study variability.

The prolonged ECT and aPTT absolute clotting times seen in children aged $<1$ year relative to adult volunteers and adult patients (in the graphical analysis) were already present at baseline and were driven by the prolonged clotting times of those aged $<2$ months (as demonstrated in the modelling analysis). Differences in baseline aPTT values may be expected as aPTT is known to be prolonged in healthy children aged $<6$ months compared with older children and adults, potentially due to decreased concentrations of coagulation factors, such as factor IX, factor XI and factor XII. ${ }^{14}$ The dTT assay employs a dilution of the paediatric plasma sample with normal adult plasma (1:8) prior to measuring dTT; this ameliorates any variation in haemostatic protein levels in the test plasma sample and, therefore, any prolongation of clotting time in the dTT would be directly correlated with the concentration of dabigatran in the plasma. This 
methodology was considered appropriate given that the purpose of the dTT assay was to provide an estimate of the concentration of dabigatran, rather than to determine the effect of age on the anticoagulant effect of dabigatran.

Results of the modelling analysis indicated that subjects with higher baseline aPTT and ECT values tended to show increased sensitivity to dabigatran exposure, which would suggest that concentration-clotting time relationships shift proportionally to a given increase or decrease in baseline value. This may explain why concentration-clotting time relationships were similar across all ages when clotting times were transformed to ratios over baseline in the graphical analysis.

The anticoagulants most commonly used in children are LMWHs, UFH and VKAs. ${ }^{4-6}$ For anticoagulation monitoring, aPTT or anti-factor (FXa) assays are usually used to measure the anticoagulant effects of UFH, anti-FXa assays are used for LMWH and the international normalized ratio (INR) is used for warfarin. ${ }^{6}$ Coagulation responses to these agents as measured by standard assays have been shown to differ according to age. ${ }^{15-17,28,29}$ For example, age-related differences in the effect and concentration of UFH, assessed using anti-FXa, anti-FIla and protamine titration assays, were demonstrated using blood samples collected from children who had received a single bolus of UFH. ${ }^{29}$ Age-based differences in coagulation responses to UFH, LMWH and warfarin have also been demonstrated using thrombin generation assays. $^{16,17}$ No such differences were observed for LMWH using thrombin clotting time and anti-FXa assays. ${ }^{30}$ The suitability of using coagulation assays designed for adults in the paediatric population must be carefully considered to avoid inappropriate clinical use.

Dietrich et al performed an in vitro study to evaluate five coagulation assays (pro-thrombin time [PT], thrombin time [TT], aPTT, dTT and ECT) for dabigatran in the paediatric population, using pooled plasma samples from healthy children. ${ }^{18}$ As seen in adults, PT (which was represented as INR) showed a linear relationship to dabigatran concentration but was relatively insensitive. aPTT was moderately sensitive but showed a non-linear concentration-response relationship, whereas TT and ECT showed linear response curves, but were overly sensitive. As in our study, aPTT and ECT were prolonged at baseline in paediatric patients compared with adults. However, no difference was seen once baseline was corrected for by calculating as a ratio over baseline. Dietrich et al found dTT to be linearly correlated with dose and the relationship was consistent across the paediatric age groups and in adults. ${ }^{18}$

Consistent with adult and in vitro paediatric data, findings from our study suggest that dTT may be the most appropriate assay for dabigatran. However, due to the relatively small number of patients, results from further paediatric clinical trials assessing dTT response with clinical outcomes will be needed to confirm this finding.

Additional clinical studies of DE in paediatric patients are underway and will provide further data regarding PK-PD relationships. The efficacy and safety of $\mathrm{DE}$ is being assessed in DIVERSITY (1160.106; NCT01895777), an on-going phase IIb/III, open-label, randomized, parallel-group study in patients aged 0 to < 18 years with VTE. Study 1160.108 (NCT02197416) is an on-going phase III, open-label, single-arm trial that will assess the safety and PK-PD of DE for the secondary prevention of VTE in children aged 0 to $<18$ years.

\section{Limitations of the Study}

The panel of clotting assays used in the paediatric studies for the current analysis was limited to dTT, ECT and aPTT. Experience with these assays was gained in the adult VTE development program. An evaluation of the similarity of concentration-effect (or PK/PD) relationships in adult versus paediatric populations is recommended as part of the paediatric drug development strategy. ${ }^{31}$ The adult comparator studies used in this analysis differ from the paediatric studies both in terms of underlying conditions of the populations and in terms of study designs. However, the selected comparator studies were deemed useful in determining the extent of similarity between paediatric and adult concentration-effect relationships.

The studies in adult volunteers were well-controlled early clinical phase studies that employed dense sampling strategies for all three selected clotting assays, allowing for precise characterization of model parameters (-Supplementary Tables S1-53, available in the online version), including baseline values for the assays. The low level of variability between subjects allowed conservative lower and upper boundaries for adult reference clotting parameters to be determined, which could be compared against paediatric parameter values (- Supplementary Fig. S1, available in the online version). Of note, healthy adult data have been used previously to demonstrate similarity of adult versus paediatric concentration-aPTT relationships. ${ }^{27}$

RE-COVER was selected as the main adult patient comparator study because it enrolled patients with objectively diagnosed VTE, as in the paediatric studies. For the graphical analyses, adult patient data from the RE-NOVATE II study were needed to supplement that from RE-COVER, due to the unavailability of dTT and baseline measurements; therefore, random betweenstudy variability may be at least in part responsible for differences in baseline and effect parameters between age groups.

It was not assessed whether the correlation between baseline clotting time (intercept) and sensitivity of the assays (slope) differs between paediatric age groups. Finally, the differences in concentration-clotting time relationship observed between adults and those aged $<2$ months is based on very few paediatric patients $(n=4)$, limiting the extent to which data can be interpreted in this age group.

\section{Conclusion}

The effects of DE on dTT were similar across age groups in both the graphical and modelling analyses. The relationship between concentration and aPTT or ECT clotting times was similar in children and adults when based on ratios over baseline in the graphical analysis. However, when absolute clotting times were used, aPTT and ECT values were slightly increased in those aged $<1$ year; modelling showed an increase in aPTT and ECT clotting times by 10 to $20 \%$ and 10 to $15 \%$, respectively, in those aged $<2$ months compared 
with adult populations. The similarity of relationships observed across paediatric age groups implies that developmental changes in the haemostatic system may have little effect on response to dabigatran, with the possible exception of children aged $<1$ year. Potentially other clinical factors currently not yet identified may introduce further variability on the response to dabigatran. The comparable relationship seen in children and adults may suggest that a similar clinical response can be expected in these two populations. However, further studies on a larger number of subjects, including neonates, are needed to confirm these findings.

\section{What is known about this topic?}

- Throughout childhood, physiological age-related changes occur in the haemostatic and coagulation systems that alter coagulation assay responses to standard anticoagulant treatments such as heparins.

- The anticoagulant effect of dabigatran across paediatric age groups had been assessed in an in vitro study that used pooled plasma samples from healthy children. When corrected for baseline, the clotting time responses to dabigatran in children were comparable between all age groups and were also similar to those in adults. There is only scarce information available from pediatric patients being treated for venous thromboembolism regarding the relationship between plasma dabigatran concentration and coagulation assay results. The current post hoc analysis was conducted to evaluate the effect of age on the relationship between dabigatran concentration and clotting time responses.

\section{What does this paper add?}

- The concentration-dTT relationships were consistent in children across all ages and adults in the graphical analysis. For ECT and aPTT, relationships based on ratios over baseline were similar across all ages; absolute clotting times showed that the same exposure resulted in longer clotting times in some of the children aged $<$ 1 year versus adults. Modelling showed concentrationclotting time relationships for all three assays were largely comparable between adults and children, except in those aged $<2$ months, in whom there was a slight upward shift in ECT and aPTT relative to adults.

- The similarity of pharmacokinetic-pharmacodynamic relationships observed across paediatric age groups implies that developmental changes in the haemostatic system may have little effect on response to dabigatran.

\section{Funding}

The study was funded by Boehringer Ingelheim International GmbH. Medical writing assistance was provided by Debbie Sherwood of PAREXEL, with funding from Boehringer Ingelheim.
Conflict of Interest

Hugo Maas, Savion Gropper, Fenglei Huang, Joachim Stangier, Igor Tartakovsky and Martina Brueckmann are employees of Boehringer Ingelheim. Jacqueline M. L Halton is a member of a Pediatric Expert Working Group for Boehringer Ingelheim. Lesley G. Mitchell is a consultant for Boehringer Ingelheim, Pfizer and Bristol-Myers Squibb.

\section{References}

1 Andrew M, David M, Adams M, et al. Venous thromboembolic complications (VTE) in children: first analyses of the Canadian Registry of VTE. Blood 1994;83(05):1251-1257

2 van Ommen $\mathrm{CH}$, Heijboer H, Büller HR, Hirasing RA, Heijmans HS, Peters M. Venous thromboembolism in childhood: a prospective twoyear registry in The Netherlands. J Pediatr 2001;139(05):676-681

3 Raffini L, Huang YS, Witmer C, Feudtner C. Dramatic increase in venous thromboembolism in children's hospitals in the United States from 2001 to 2007. Pediatrics 2009;124(04):1001-1008

4 Parasuraman S, Goldhaber SZ. Venous thromboembolism in children. Circulation 2006;113(02):e12-e16

5 Bonduel MM. Oral anticoagulation therapy in children. Thromb Res 2006;118(01):85-94

6 Monagle P, Chan AK, Goldenberg NA, et al. Antithrombotic therapy in neonates and children: Antithrombotic Therapy and Prevention of Thrombosis, 9th ed: American College of Chest Physicians EvidenceBased Clinical Practice Guidelines. Chest 2012;141:e737S-e801S

7 Chan AK, Monagle P. Updates in thrombosis in pediatrics: where are we after 20 years? Hematology (Am Soc Hematol Educ Program) 2012;2012:439-443

8 Schulman S, Kearon C, Kakkar AK, et al; RE-COVER Study Group. Dabigatran versus warfarin in the treatment of acute venous thromboembolism. N Engl J Med 2009;361(24):2342-2352

9 Schulman S, Kearon C, Kakkar AK, et al; RE-MEDY Trial Investigators; RE-SONATE Trial Investigators. Extended use of dabigatran, warfarin, or placebo in venous thromboembolism. $\mathrm{N}$ Engl J Med 2013;368(08):709-718

10 Schulman S, Kakkar AK, Goldhaber SZ, et al; RE-COVER II Trial Investigators. Treatment of acute venous thromboembolism with dabigatran or warfarin and pooled analysis. Circulation 2014;129 (07):764-772

11 Kuhle S, Eulmesekian P, Kavanagh B, et al. Lack of correlation between heparin dose and standard clinical monitoring tests in treatment with unfractionated heparin in critically ill children. Haematologica 2007;92(04):554-557

12 Mitchell LG, Vegh P. Conventional chromogenic heparin assays are influenced by patient's endogenous plasma antithrombin levels. Klin Padiatr 2010;222(03):164-167

13 Kuhle S, Male C, Mitchell L. Developmental hemostasis: pro- and anticoagulant systems during childhood. Semin Thromb Hemost 2003;29(04):329-338

14 Appel IM, Grimminck B, Geerts J, Stigter R, Cnossen MH, Beishuizen A. Age dependency of coagulation parameters during childhood and puberty. J Thromb Haemost 2012;10(11):2254-2263

15 Ignjatovic V, Furmedge J, Newall F, et al. Age-related differences in heparin response. Thromb Res 2006;118(06):741-745

16 Chan AK, Berry LR, Monagle PT, Andrew M. Decreased concentrations of heparinoids are required to inhibit thrombin generation in plasma from newborns and children compared to plasma from adults due to reduced thrombin potential. Thromb Haemost 2002;87(04):606-613

17 Massicotte P, Leaker M, Marzinotto V, et al. Enhanced thrombin regulation during warfarin therapy in children compared to adults. Thromb Haemost 1998;80(04):570-574

18 Dietrich K, Stang L, van Ryn J, Mitchell LG. Assessing the anticoagulant effect of dabigatran in children: an in vitro study. Thromb Res 2015;135(04):630-635 
19 Halton JML, Albisetti M, Luciani M, et al. Pharmacokinetics and pharmacodynamics of a single-dose oral solution of dabigatran given after standard anticoagulation therapy in children, 2-12 years old, with venous thromboembolism. ISTH SCC, May 25-28, 2016

20 Halton JML, Lehr T, Cronin L, et al. Safety, tolerability and clinical pharmacology of dabigatran etexilate in adolescents. An openlabel phase IIa study. Thromb Haemost 2016;116(03):461-471

21 Halton JML, Albisetti M, Biss B, et al. Phase Ila study of dabigatran etexilate in children with venous thrombosis: pharmacokinetics, safety, and tolerability. J Thromb Haemost 2017;15(11):2147-2157

22 Halton JML, Picard AC, Harper R, et al. Pharmacokinetics, pharmacodynamics, safety and tolerability of dabigatran etexilate oral liquid formulation in infants with venous thromboembolism. Thromb Haemost 2017;117(11):2168-2175

23 Glund S, Stangier J, Schmohl M, et al. Safety, tolerability, and efficacy of idarucizumab for the reversal of the anticoagulant effect of dabigatran in healthy male volunteers: a randomised, placebo-controlled, double-blind phase 1 trial. Lancet 2015;386(9994):680-690

24 Glund S, Stangier J, van Ryn J, et al. Effect of age and renal function on idarucizumab pharmacokinetics and idarucizumab-mediated reversal of dabigatran anticoagulant activity in a randomized, double-blind, crossover phase Ib study. Clin Pharmacokinet 2017;56(01):41-54
25 Eriksson BI, Dahl OE, Huo MH, et al; RE-NOVATE II Study Group. Oral dabigatran versus enoxaparin for thromboprophylaxis after primary total hip arthroplasty (RE-NOVATE II*). A randomised, double-blind, non-inferiority trial. Thromb Haemost 2011;105(04):721-729

26 Upton RN, Mould DR. Basic concepts in population modelling, simulation, and model-based drug development: part 3-introduction to pharmacodynamic modeling methods. CPT Pharmacometrics Syst Pharmacol 2014;3:e88

27 Madabushi R, Cox DS, Hossain M, et al. Pharmacokinetic and pharmacodynamic basis for effective argatroban dosing in pediatrics. J Clin Pharmacol 2011;51(01):19-28

28 Hanslik A, Kitzmüller E, Tran US, et al. Monitoring unfractionated heparin in children: a parallel-cohort randomized controlled trial comparing 2 dose protocols. Blood 2015;126(18):2091-2097

29 Newall F, Ignjatovic V, Johnston L, et al. Age is a determinant factor for measures of concentration and effect in children requiring unfractionated heparin. Thromb Haemost 2010;103(05):1085-1090

30 Ignjatovic V, Summerhayes R, Newall F, Monagle P. The in vitro response to low-molecular-weight heparin is not age-dependent in children. Thromb Haemost 2010;103(04):855-856

31 European Medicines Agency. Reflection paper on the use of extrapolation in the development of medicines for paediatrics. 09 October 2017; EMA/199678/2016 